\title{
Evaluation of the Effect of Training on Unstable Ground on Dynamic Stability in Soccer Players Using the Y-Balance Test
}

\author{
Paweł Kaczmarczyk ${ }^{\mathbf{1}}$, Paweł Ryngier ${ }^{\mathbf{1}}$ \\ ${ }^{1}$ Faculty of Physiotherapy, Academy of Physical Education in Katowice \\ Correspondence to: Paweł Ryngier, \\ Faculty of Physiotherapy, Academy of Physical Education in Katowice, Mikołowska 72A, 40-065 Katowice, Poland, \\ p.ryngier@awf.katowice.pl
}

DOI: https://doi.org/10.5114/phr.2021.104601

Received: 31.01.2021 Reviewed: 01.02.2021 Accepted: 07.02.2021

\begin{abstract}
Background: The aim of this study was to analyse the effect of training on unstable ground on dynamic stability as measured by the Y-Balance Test (YBT).

Materials and methods: The study was conducted on 16-year-old football players from the 'Zagłębie Sosnowiec' club in Poland. The YBT, which is a dynamic test that assesses strength, flexibility and coordination, was used as a research tool. For this purpose, a ' $\mathrm{Y}$ ' shape was drawn on flat ground, and each component line was given a separate name: anterior (A), posterolateral (PL) and posteromedial (PM). The training on unstable ground lasted 6 weeks. Each study group performed 2 training sessions just before the regular football training at the club. The control group received only football training conducted by the club coaches. Throughout this period, 12 central stabilization training sessions were conducted for the study group.
\end{abstract}

Results: At the completion of the project, significant progress was observed in the YBT for the study group compared to the control group. In the A reach direction for the left lower limb, a statistically significant improvement was documen- ted in the study group in the second test $(\mathrm{p}<0.05)$. In the PM direction, the second test also revealed a statistically significant improvement for the study group $(\mathrm{p}<0.05)$. Finally, the PL distance reached by the study group in the second test showed a statistically significant increase compared to the previous test for both the right and left lower limbs $(\mathrm{p}<0.05)$.

Conclusions: Core stability training on unstable ground improves performance in the YBT for the right lower limb in the PM and PL directions and for the left lower limb in the A, PM and PL reach directions. In both the study and control groups, there were no significant differences (asymmetries) in the A reach direction nor in the composite score between the right and left lower limbs.

\section{Key words}

football, training, dynamic stabilisation, Y-Balance Test 


\section{Introduction}

NAlong with advances in the science of stabilisation and the growing interest in the field, people have begun to wonder about the significance stabilisation might have for various sports disciplines. Undoubtedly, all athletes with outstanding technique perform the movement activities that their discipline requires in a perfect manner. Elphinston [1] proposed a simple, useful definition for functional stability in sport: 'the ability to use individual body structures in the safest, most effective positions possible, with regard to the functional tasks being imposed on them' [1]. A few years later, the definition of this concept was revised: functional stability means the ability to use strength and endurance in a functional manner in all planes of movement, despite changes in the location of the body's centre of gravity (COG).

It is worth mentioning that good postural control and core stabilisation, for both coach and athlete, can be identified with an impeccable technique in many cases. For example, a good swimmer uses water resistance to push off effectively and thus move efficiently. However, without proper stabilisation of the trunk and glenohumeral joint, this same resistance could drive the shoulder into an unfavourable position. This would result in incorrect movement biomechanics, and as a consequence, the swimmer could injure a shoulder joint [2].

Therefore, in the author's opinion, the safety of the movement and its efficiency are the most important factors in this context. Most often, in the initial phase of training, a lot of emphasis is placed on the technical skills of the particular sport discipline; certain actions that are elements of technique may involve muscles to an excessive degree or in a sequence that is not entirely correct. The result is a lack of movement fluidity and confidence, which requires much more effort than in the case of a more experienced athlete in the same sport [3, 4].

Professional athletes use only the muscles they need for a given performance. This allows them to perform specific activities longer, more precisely, more safely (without compensation) and without wasting unnecessary energy. Functional stabilisation improves technique by increasing the biomechanical efficiency of movement. It can also reduce the risk of injury by minimising overload on the structures concerned and providing appropriate control to perform the activity more safely. In a situation of lack or reduced joint stability, the force used to perform a given action is dispersed and not fully utilised. This phenomenon is not observed when a given movement segment is stabilised, and the movement becomes more effective due to this. Therefore, the effect is adequate to the effort involved. Functional stability, as the ability to move or hold a given position in a safe, controlled and fully effective way, is extremely important in football as well $[1,5,6]$. Besier et al. [7] reported that multi-disciplinary athletes are injured because they are unable to control their balance and COG during sudden accelerations, decelerations and changes in movement directions. Football players often change direction and have to perform many movements while extremely unbalanced. A better control of the body provides the athlete with many advantages; therefore, the interest in the topic of stabilisation among managers and coaches of motor preparation is fully understandable. Many of the best football teams in the world pay special attention to the elements of stabilisation training. As described previously, this helps to reduce the risk of injury, as well as increase efficiency during competition, which can undoubtedly determine the results of a single match, round or season.

\section{Aims}

The aim of this study was to examine the effect of training on unstable ground on dynamic stability parameters assessed using the Y-Balance Test (YBT). 


\section{Materials and Methods}

The study was conducted among 16-year-old football players of the 'Zagłębie Sosnowiec' club in Poland. Twenty-four players were randomly assigned to particular groups $(n=12$ to the study group and $\mathrm{n}=12$ to the control group). The baseline characteristics of the study subjects, such as body mass and height, are presented in Table 1.

Table 1. A detailed characteristic of the study subjects.

\begin{tabular}{|c|c|c|c|c|c|c|c|}
\hline \multirow{2}{*}{ Subjects } & \multirow{2}{*}{ Age (years) } & \multicolumn{3}{|c|}{ Body height (cm) } & \multicolumn{3}{c|}{ Body mass (kg) } \\
\cline { 3 - 8 } & & $\overline{\mathrm{x}}$ & $\begin{array}{c}\text { Min } \\
\text { Max }\end{array}$ & SD & $\overline{\mathrm{x}}$ & Min & SD \\
& & 16 & 164 & 6.11 & 64.2 & 51.7 & 7.88 \\
\hline Men & 16 & 188 & & & 81.2 & \\
$(\mathrm{n}=24)$ & & & & & &
\end{tabular}

Legend: $\mathrm{n}$ - number of participants, $\overline{\mathrm{x}}$ - mean, SD - standard deviation, Min - minimum, Max - maximum.

Before the study began, the players, coaching staff and club authorities agreed to participate. They were also informed about the possibility of withdrawal at any time during its course and about the research objectives and methods that will be used.

Inclusion criteria: (1) 16-year-old players at the 'Zagłębie Sosnowiec' club; (2) no serious injuries in the last 3 months; (3) the ability to perform all tests and exercises correctly; and (4) consent from the players, their legal guardians, coaches and the club authorities for the subjects to participate in the study. Exclusion criteria: (1) player withdrawal from the study; (2) injury, illness or other conditions precluding further training or testing; and (3) absence from training sessions. Two players were excluded from the study group, while four players were excluded from the control group. The main reasons were injuries or player loans to other clubs (absence from training sessions and/or final tests).

The YBT was used as a research tool in this study. This is a dynamic test which requires the test subject to have strength, flexibility and good coordination. It allows for determining the risk of lower limb injury and detecting any chronic instability of the ankle joint. The YBT can also reveal functional asymmetries between the left and right sides of the body and can be used to check the athlete's preparation to return to sport after injury.

Prior to the start of the study, all participants were introduced to the principles of the above test. An instructional demonstration was also performed to visualise the task. Each subject was given a pre-test of a motor task. The YBT was performed with bare feet or socks. To conduct the YBT, a Y symbol was drawn on a flat floor using wide, non-transparent adhesive tape and tailor's tape attached to it, maintaining the appropriate lengths of and the angles between the lines. Each line was labelled: anterior (A), posterolateral (PL), and posteromedial (PM). Figure 1 shows the line diagram for the Y- Balance Test when testing the right lower limb (A) and the left lower limb (B). 


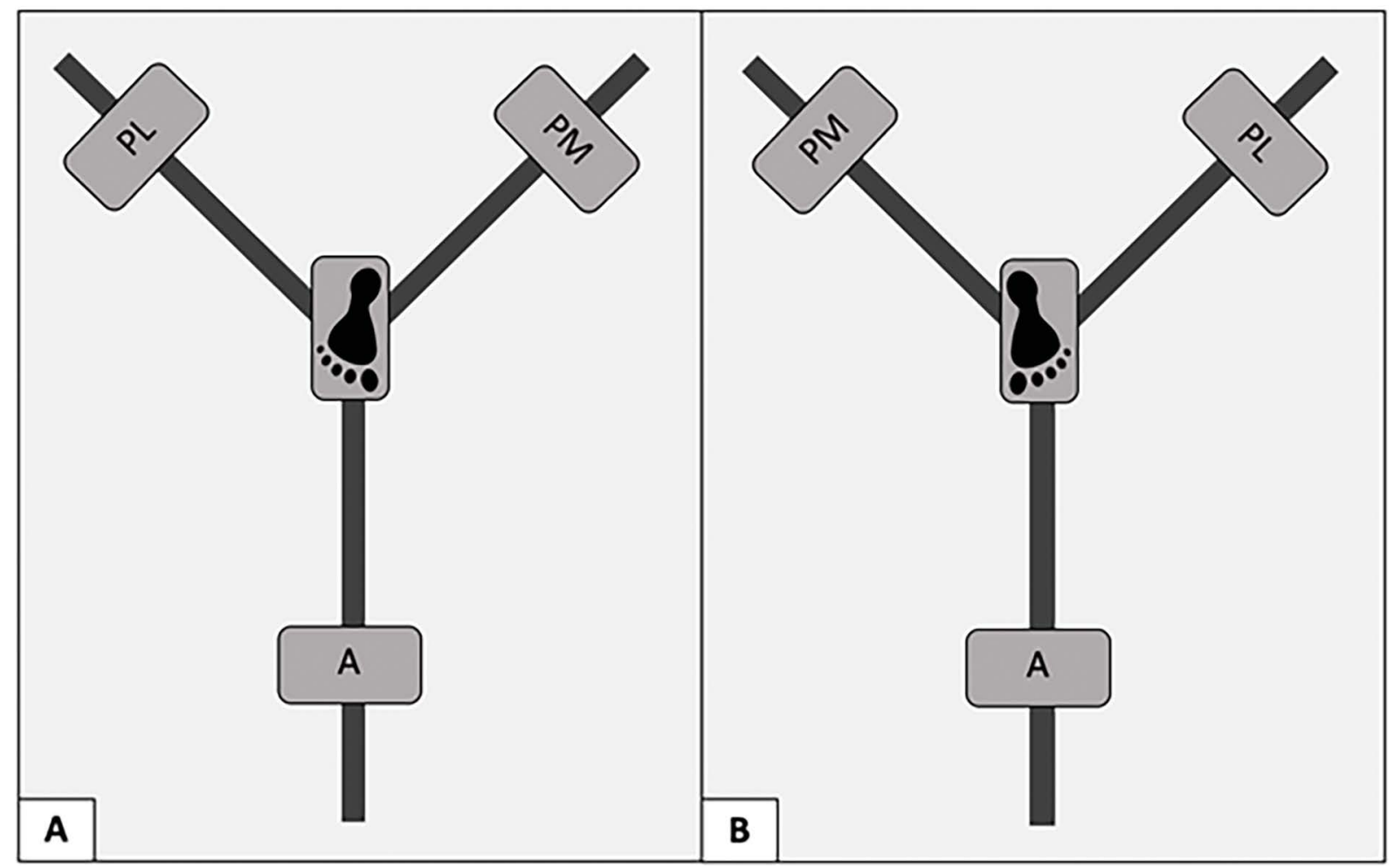

Figure 1. Y-Balance Test: line diagram for the right (A) and left lower limb (B).

Legend: A - anterior, PL - posterolateral, PM - posteromedial.

The angle between line A and both PM and PL was $135^{\circ}$. In turn, the angle between PM and PL was $90^{\circ}$. The length of each line was equal to $150 \mathrm{~cm}$. The foot of the tested limb was placed in the middle of the Y symbol. The test was to reach as far as possible along each of the component lines with the toes of the other lower limb (unsupported). The test was marked as not completed or had to be repeated if: (1) the 'touch' was at the same time a transfer of body weight to the reaching lower limb, thus providing an additional point of support; (2) the foot of the standing limb separated from or slid across the floor; or (3) there was a loss of balance while reaching or returning to the starting position.

Each subject performed 3 repetitions of the test, and the best result was taken for analysis. The subject was allowed to rest in a standing position with both feet for up to $30 \mathrm{~s}$ after each test and up to 1 min after completing the entire test. A participant could also voluntarily restart the test ear- lier. The distances obtained were measured in $\mathrm{cm}$ directly during the test. Each time the test was completed, the results were read out and recorded on test sheets. It is extremely important to note that each result was normalized by dividing the obtained score by the length of the lower limb measured from the anterior superior iliac spine to the lateral ankle.

The period of training on unstable ground for the study group lasted for 6 weeks, with 2 training sessions each (12 sessions in total), performed just before the standard football training. The control group received only football training. The following were used for core stability training: Swiss balls, sensomotoric discs, gymnastic ladders, mattresses, hurdles and footballs. The exercises were strictly defined and individualised to the subject's possibilities, to be able to correctly perform each repetition without any compensation. The exercises ranged from their easiest form (difficulty level 1) to their hardest form (difficulty 
level 3). The athlete could attempt to perform an exercise from a higher level if he performed the lower level correctly. Three of the four exercises were static, involving holding a particular body posture for a few seconds. The fourth exercise was dynamic in nature by using a football.

All data acquired were collected as a database in Statistica 10 (Dell Inc., Tulsa, USA). Descriptive statistics tools were used, and analysis of variance with repeated measures was performed. The statistical analyses used the standardised results for each trial (the A, PM and PL directions) and each lower limb separately. This means that the final result is closely related to the length of the test subject's lower limb.

\section{Results}

The mean lower limb length was $95.8 \pm 4.3 \mathrm{~cm}$ in the study group and $96.9 \pm 2.9 \mathrm{~cm}$ in the control group. The mean results with standard deviations for both groups and each lower limb separately are shown in Table 2. A composite score (CS) was also calculated as the arithmetic mean of the 3 standardised scores according to the formula:

$$
C S=(A+P M+P L) / 3
$$

where the following symbols were used:

A - anterior,

PL - posterolateral,

PM - posteromedial.

Table 2. Results from both comparison groups for the right and left lower limb.

\begin{tabular}{|c|c|c|c|c|}
\hline \multicolumn{5}{|c|}{ Right lower limb } \\
\hline \multirow{2}{*}{ Performed test } & \multicolumn{2}{|c|}{ Control group } & \multicolumn{2}{|c|}{ Study group } \\
\hline & $1^{\text {st }}$ test & $2^{\text {nd }}$ test & $1^{\text {st }}$ test & $2^{\text {nd }}$ test \\
\hline $\mathrm{A}(\overline{\mathrm{x}} \pm \mathrm{SD})$ & $74 \pm 5.5$ & $75 \pm 5.7$ & $74 \pm 4.9$ & $76 \pm 5.5$ \\
\hline $\mathrm{PM}(\overline{\mathrm{x}} \pm \mathrm{SD})$ & $117 \pm 6.1$ & $119 \pm 6.2$ & $115 \pm 6.8$ & $124 \pm 6.2$ \\
\hline $\mathrm{PL}(\overline{\mathrm{x}} \pm \mathrm{SD})$ & $116 \pm 5.7$ & $117 \pm 5.6$ & $112 \pm 6.8$ & $120 \pm 5.5$ \\
\hline $\mathrm{CS}(\overline{\mathrm{x}} \pm \mathrm{SD})$ & $103 \pm 4.96$ & $104 \pm 5.43$ & $100 \pm 5.10$ & $107 \pm 4.46$ \\
\hline \multicolumn{5}{|c|}{ Left lower limb } \\
\hline \multirow{2}{*}{ Performed test } & \multicolumn{2}{|c|}{ Control group } & \multicolumn{2}{|c|}{ Study group } \\
\hline & $1^{\text {st }}$ test & $2^{\text {nd }}$ test & $1^{\text {st }}$ test & $2^{\text {nd }}$ test \\
\hline $\mathrm{A}(\overline{\mathrm{x}} \pm \mathrm{SD})$ & $74 \pm 4.6$ & $75 \pm 5.3$ & $75 \pm 4.5$ & $77 \pm 4.8$ \\
\hline $\mathrm{PM}(\overline{\mathrm{x}} \pm \mathrm{SD})$ & $118 \pm 6.3$ & $120 \pm 5.2$ & $116 \pm 8.9$ & $124 \pm 6.2$ \\
\hline $\operatorname{PL}(\overline{\mathrm{x}} \pm \mathrm{SD})$ & $115 \pm 5.7$ & $116 \pm 5.5$ & $113 \pm 7.6$ & $121 \pm 5.9$ \\
\hline $\mathrm{CS}(\overline{\mathrm{x}} \pm \mathrm{SD})$ & $102 \pm 4.69$ & $104 \pm 4.59$ & $101 \pm 5.06$ & $108 \pm 4.17$ \\
\hline
\end{tabular}

Legend: A - anterior, PL - posterolateral, PM - posteromedial, CS - composite score, $\overline{\mathrm{x}}$ - mean, SD - standard deviation. 
During the first test in the A direction with the left lower limb, there was no significant increase in the differences for the two groups. The study group presented a slightly lower level than the control group in the first test. The second test documented slightly better results for the study group, but the results were not statistically significant ( $p>0.05)$.

During the first test in the A reach direction with the right lower limb, a statistically significant improvement in the study group was documented $(\mathrm{p}<0.05)$. The study group appeared to perform better than the control group in both tests. In the first test, the differences between the groups were very slight, with an advantage for the study group. The second test showed a statistically significant increase in the distances reached by the study group $(\mathrm{p}<0.05)$, an effect not observed in the control group. In both the first and second tests, the control group remained at almost the same level.

In the test along the PM direction, statistically significant differences were observed in the distances reached by the study group compared to the control group. In the first test, greater distances were reached by the control group, both for the right and left lower limb. The second test showed a statistically significant improvement for the study group $(\mathrm{p}<0.05)$ for both lower limbs in comparison to the previous results. There were also better results for the study group than for the control group.

The last of the 3 tests was along the PL direction. In the first test, the control group achieved better results than the study group for both limbs. In the second test, a statistically significant increase in the distances achieved by the study group was observed compared to the previous test $(p<0.05)$, for both lower limbs. There were also better results in the study group for both lower limbs compared to the control group.

\section{Discussion}

The positive effect of stabilisation training obtained in this study is confirmed by previous rese- arch reports of YBT distances increasing after a training period that included stabilisation exercises. In 2010, Filipa et al. [8] observed an increase in CS parameter on the Star Excursion Balance Test (SEBT) in the study group, which performed stabilisation exercises alternated with strength exercises (e.g., barbell squats, jumping jacks, forward bridges and others using unstable ground) for 8 weeks. Similarly to our study, the control group remained at a similar level in both tests in terms of the sum of all 3 scores for both lower limbs (CS). In contrast, the study group achieved a statistically significant improvement $(p<0.03)$ when comparing pre- and post-training results. It is interesting how much strength training alone, without any elements of unstable ground, can affect YBT results.

Another study by Colado et al. [9] revealed a significant advantage in terms of the degree of trunk muscle recruitment for the deadlift with load compared to other types of popular exercises, some of which on unstable ground. Perhaps future studies will pay more attention to this issue and investigate whether strength training, including exercises such as deadlifts or barbell squats, has a significant effect on dynamic stability.

Nevertheless, the results noted in this work should be treated with some caution. The study group was relatively small to provide solid scientific evidence, so further studies on a larger population are recommended. At the same time, over the last few years, research papers have focused on the ambiguous effect of exercise on unstable ground on dynamic stability. Some have reported no significant difference between training on unstable ground vs. standard training. In the study cited above, the only differentiating element was the use of unstable ground in the study group, which, as it turned out, did not bring any significant changes in the results of functional tests (SEBT) [10]. Importantly, the differentiation of study groups by age, gender and type of sport may be valid in this case and affect the effectiveness of training on unstable ground.

Nowadays, in the field of sports all over the world, it is very easy to notice that it often takes extreme 
efforts to join the elite. Very high training loads, which are supposed to lead an athlete to achieve outstanding results in a particular discipline, often result in various types of injuries. Analysing the findings presented in this paper and those of other researchers, it seems reasonable to implement stabilisation exercises as a kind of prevention from various sports injuries. Increasing the level of dynamic stability reduces the risk of injury and gives the athlete better body control. In addition, in pre-season, it seems advisable to perform the YBT, which can provide an early indication of possible functional deficits. This test also allows to efficiently monitor the progress of rehabilitation if an injury occurs. An additional advantage of the YBT is that it is an easy and highly reliable methodology, which has been extensively studied and is highly demanded nowadays.

\section{Conclusions}

Core stability training on unstable ground improves the results obtained in the YBT for the right lower limb in the PM and PL directions and for the left lower limb in the A, PM and PL reach directions. There were no significant differences (asymmetries) in the A and CS distances between the right and left lower limbs for either the study or control group.

\section{References}

1. Elphinston J. Stability, Sport and Performance Movement. Great technique without injury, North Atlantic Books, 2008.

2. Heinlein SA, Cosgarea AJ. Biomechanical Considerations in the Competitive Swimmer's Shoulder. Sports Health. 2010; 2 (6): 519-525.

3. Sharrock C, Cropper J, Mostad J, Johnson M, Malone T. A pilot study of core stability and athletic performance: is there a relationship? Int J Sports Phys Ther. 2011; 6 (2): 63-74.

4. Clark DR, Lambert MI, Hunter AM. Contemporary perspectives of core stability training for dynamic athletic performance: a survey of athletes, coaches, sports science and sports medicine practitioners. Sports Med Open. 2018; 4 (1): 32.

5. Young WB. Transfer of strength and power training to sports performance. Int J Sports Physiol Perform. 2006; 1 (2): 74-83.

6. Wirth K, Hartmann H, Mickel C, Szilvas E, Keiner M, Sander A. Core Stability in Athletes: A Critical Analysis of Current Guidelines. Sports Med. 2017; 47 (3): 401-414.
7. Besier TF, Lloyd DG, Ackland TR, Cochrane JL. Anticipatory effects on knee joint loading during running and cutting maneuvers. Med Sci Sports Exerc. 2001; 33 (7): 1176-1181.

8. Filipa A, Byrnes R, Paterno MV, Myer GD, Hewett TE. Neuromuscular training improves performance on the star excursion balance test in young female athletes. J Orthop Sports Phys Ther. 2010; 40 (9): 551-558.

9. Colado JC, Pablos C, Chulvi-Medrano I, Garcia-Masso X, Flandez J, Behm DG. The progression of paraspinal muscle recruitment intensity in localized and global strength training exercises is not based on instability alone. Arch Phys Med Rehabil. 2011; 92 (11): 1875-1883.

10. Granacher U, Schellbach J, Klein K, Prieske O, Baeyens JP, Muehlbauer T. Effects of core strength training using stable versus unstable surfaces on physical fitness in adolescents: a randomized controlled trial. BMC Sports Sci Med Rehabil. 2014; 6 (1): $40-46$. 